\title{
Experimental signatures of time-reversal-violating superconductors
}

\author{
Tai-Kai Ng \\ Department of Physics, Hong Kong University of Science and Technology, Clear Water Bay Road, Hong Kong \\ C. M. Varma \\ University of California, Riverside, California 92521, USA \\ (Received 23 March 2004; published 20 August 2004)
}

\begin{abstract}
We discuss some experimental signatures associated with the topological structures of unconventional superconductor order parameters of form $d_{x^{2}-y^{2}}+i x$, where $x=s, p_{x} \pm p_{y}$, or $d_{x y}$. Specifically, we study the topological surface states on the (110) and equivalent surfaces of such superconductors which are observable in Andreev tunneling experiments, as well as evaluate the magnetic flux trapped in superconducting rings of such superconductors with multiple grain-boundary Josephson junctions. Previous experiments are examined and several new experiments suggested.
\end{abstract}

DOI: 10.1103/PhysRevB.70.054514

PACS number(s): 74.20.Rp, 74.45.+c, 74.50.+r

\section{INTRODUCTION}

Definitive experiments relying on topological differences between different superconductors to reveal their symmetry are of interest in themselves. The motivation for such investigations in the cuprates is especially strong. It has been suggested that the pseudogap regime in the phase diagram of the cuprates ends at a quantum critical point and that the pseudogap regime represents an elusive time-reversalviolating symmetry. ${ }^{1}$ Symmetry-based proposals ${ }^{2}$ for experiments in the normal state using circularly polarized angle resolved photoemission experiments show results ${ }^{2,3}$ consistent with a time-reversal-violating phase in the normal and superconducting phases below a critical density.

The two time-reversal-violating normal states suggested for the underdoped cuprates have a current pattern sketched in Fig. 1 of Ref. 2. State I has two domains specified by the sign of the phase and preserves symmetry on reflections in planes normal to the $x-y$ plane going along the $x$ and $y$ axes but violates it on reflections in the $x \pm y$ planes. State II has four domains, two due to time-reversal and two because reflection symmetry is violated either over planes going through $(x+y)$ or $(x-y)$ axes. This state breaks Inversion symmetry and is the state consistent with the experiments of Kaminski et al. ${ }^{3}$ Considerations of these symmetries ${ }^{4}$ is important for the design of experiments.

Suppose such states were to undergo continuous transitions to superconductors of dominant $d_{x^{2}-y^{2}}$ symmetry. Then a time-reversal-violating component would be linearly admixed which by continuity preserves the reflection symmetry of the normal state. For state I, such an admixed state is uniquely of the $d_{x y}$ variety giving a $d_{x^{2}-y^{2}} \pm i d_{x y}$ superconductor; the \pm sign signifies the two time-reversed domains. For state II, the admixed states possible are of the $p_{x} \pm p_{y}$ variety, giving a $d_{x^{2}-y^{2}} \pm i\left(p_{x} \pm p_{y}\right)$ superconductor. The same conclusions have been reached ${ }^{5}$ on pure group-theoretical grounds.

Time-reversal breaking through admixture of the $i s$ or $i d_{x y}$ type near surfaces due to depairing of the $d_{x^{2}-y^{2}}$ order parameter and their effect on Andreev reflection experiments have been studied. ${ }^{6}$ Our interest here is not such surface induced time-reversal breaking but time-reversal breaking in the bulk. There were proposals for other time-reversal-violating superconducting states from different considerations. ${ }^{7,8} \mathrm{We}$ consider below three types of time-reversal-violating superconducting states, $d_{x^{2}-y^{2}}+i x\left[x=s, d_{x y}\right.$ or $\left.p_{x} \pm p_{y}\right]$, with small imaginary component $i x$. Where the context is clear, let us call them $d+i s, d+i d, d+i p$.

The magnitude of the mixed order parameter is believed to be small. In the case of $d+i p$ state, the experiment of Kaminski et al. ${ }^{3}$ indicated that the gauge-invariant imaginary part of the wave function is about $5 \%$ of the real part. This is also the estimate we should have for the magnitude of the $p$ wave order in relation to the dominant $d$ order. The smallest of the mixed imaginary order parameter implies that the admixture is difficult to be detected by quantitative measurements. Instead, we should consider experiments that show qualitative differences in the presence of admixture order parameter. Two kinds of experiments that satisfy this requirement will be discussed here. The experimental signatures reflect the topological structures of the order parameters and are robust to small perturbations. The first experiment discussed is an Andreev-type tunneling experiment on the (110) (and equivalent) surfaces of the high- $T_{c}$ cuprates.

\section{SURFACE STATES ON (110) SURFACES}

The presence of surface state with energy $\epsilon=0$ on the (110) (and equivalent) surfaces of (pure) $d_{x^{2}-y^{2} \text {-wave super- }}$ conductors was first pointed out by $\mathrm{Hu}^{9}$ We extend Hu's analysis here to study surface states in superconductors with small deviation from the pure $d_{x^{2}-y^{2}}$ symmetry. Following Hu's notation, we work with a coordinate system where the $x$ and $y$ axes are the $(1 \overline{1} 0)$ and (110) crystal axes of the cuprates, respectively. In this notation, the order parameter of a pure $d_{x^{2}-y^{2}}$-wave superconductor has the property $\Delta\left(k_{x}, k_{y}, k_{z}\right)=-\Delta\left(-k_{x}, k_{y}, k_{z}\right) .{ }^{9}$ The electronic excitations of an inhomogeneous superconductor are determined by the Bogoliubov-de Gennes equations, 


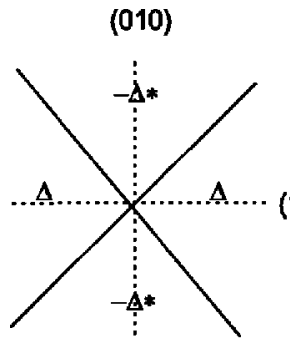

(a) d+is
(100)

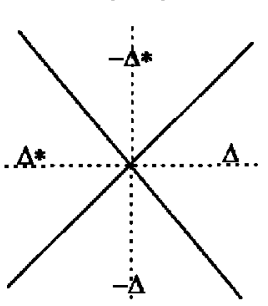

(b)

$d+i p$
(100)

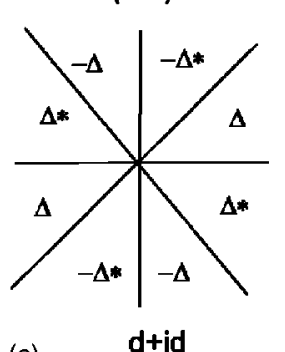

(100)

$$
\begin{gathered}
\varepsilon_{n} u_{n}(\vec{x})=\hat{H}_{o} u_{n}(\vec{x})+\int d \vec{x}^{\prime} \Delta\left(\vec{x}, \vec{x}^{\prime}\right) v_{n}\left(\vec{x}^{\prime}\right), \\
\epsilon_{n} v_{n}(\vec{x})=-\hat{H}_{o} v_{n}(\vec{x})+\int d \vec{x}^{\prime} \Delta\left(\vec{x}, \vec{x}^{\prime}\right) u_{n}\left(\vec{x}^{\prime}\right),
\end{gathered}
$$

where $\hat{H}_{0}=-1 / 2 m \nabla_{\vec{x}}^{2}-\mu+v(\vec{x}), v(\vec{x})$ is the electron crystal potential and $\Delta\left(\vec{x}, \vec{x}^{\prime}\right)$ is a general, nonlocal superconductor order parameter. A superconductor at $x>0$ with surface at $x=0$ with thickness $d$ can be modeled by a superconductor order parameter of form $\Delta(\vec{k}, \vec{x}) \sim \Delta_{0}(\vec{k}) \Theta(x)$, with boundary condition that $u(v)_{n}(\vec{x})$ vanishes at $x=-d .{ }^{9} \Delta_{0}(\vec{k})$ is the wavevector-dependent order parameter of the bulk superconductor. The Bogoliubov-de Gennes equations for electronic states close to the Fermi surface can be solved in the WKBJ approximation. The surface states with $u(v)(\vec{x})$ $\sim e^{i\left(k_{F y} y+k_{F z} z\right)} e^{-\gamma x}$ are determined by the following eigenvalue equation: ${ }^{9}$

$$
e^{4 i \epsilon\left(m / k_{F x}\right) d}=\left(\frac{\Delta_{+}}{\Delta_{-}}\right)\left(\frac{\epsilon+i \sqrt{\left|\Delta_{-}\right|^{2}-\epsilon^{2}}}{\epsilon-i \sqrt{\left|\Delta_{+}\right|^{2}-\epsilon^{2}}}\right),
$$

where $|\epsilon|<\left|\Delta_{+(-)}\right|$is the surface state energy, $\Delta_{ \pm}$ $=\Delta_{0}\left( \pm k_{F x}, k_{F y}, k_{F z}\right) . \vec{k}_{F}=k_{F x}, k_{F y}, k_{F z}$ is a wave vector on the Fermi surface.

Notice that $\epsilon$ depends on $k_{F y}, k_{F z}$ implicitly through the momentum dependence of the gap function $\Delta_{0}(\vec{k})$. For pure $d$-wave superconductors, $\Delta_{+}=-\Delta_{-}$and the eigenvalue equation has a particular solution $\epsilon=0$ that is independent of $\vec{k}_{F}$ and surface thickness $d$. This is the zero energy midgap state discovered by Hu. ${ }^{9}$ Other surface states may also exist. However they always exist in pairs with energies $\pm \epsilon$ because of particle-hole symmetry in the eigenvalue equation. Consequently in the tunneling experiment across the (110) surfaces, a "zero-bias conductance peak" is expected to be observed with a particle-hole symmetric tunneling spectrum.

In the presence of a small non- $d$-wave order parameter component the relation $\Delta_{+}=-\Delta_{-}$is modified. To begin with, let $\Delta$ be real with $\Delta_{+}=\Delta+s$ and $\Delta_{-}=-\Delta+s$, where $s$ is independent of $\vec{k}$, corresponding to the allowed symmetry in an orthorhombic crystal with time-reversal preserved. By direct inspection of Eq. (2) we see that the $\epsilon=0$ surface state survives in the presence of the small $s$ component for all momenta $k_{F y}, k_{F z}$, as long as $\Delta_{0}(\vec{k})>s$. Other surface states may also exist in pairs with energies $\pm \epsilon$ following from particle-

hole symmetry. Since the surface states energies must satisfy $|\epsilon|<\Delta_{0}(\vec{k})-s$, the main effect of mixing a real non- $d$-wave component is to reduce the number of surface states that are allowed in pure $d$-wave superconductors. Consequently tunneling experiment cannot distinguish effectively pure $d$-wave superconductors from superconductors mixed with a real, non- $d$-wave component.

The situation changes if we consider superconductors with complex order parameter satisfying $\Delta_{+}=-\Delta_{-}^{*}$. In this case the eigenvalue equation becomes

$$
e^{4 i \epsilon\left(m / k_{F x}\right) d}=-e^{2 i \phi}\left(\frac{\epsilon+i \sqrt{\left|\Delta_{0}\right|^{2}-\epsilon^{2}}}{\epsilon-i \sqrt{\left|\Delta_{0}\right|^{2}-\epsilon^{2}}}\right)^{2},
$$

$\phi$ is defined through $\Delta_{ \pm}=\left|\Delta_{0}\right| e^{ \pm i \phi}$. The \pm signs in front of $\phi$ correspond to opposite domains of time reversal. Equation (3) has the formal solution $\left(2 m d / k_{F x}\right) \epsilon-\phi$ $=-\tan ^{-1}\left(\epsilon / \sqrt{\left|\Delta_{0}\right|^{2}-\epsilon^{2}}\right)$. Notice that for $\phi \neq 0 \epsilon=0$ state is no longer a solution to the equation. Writing $\epsilon=\left|\Delta_{0}\right| \sin \theta$ we find that $\theta=\phi$ when $d=0$ and the surface state energy measures the imaginary part of the gap function $\left|\Delta_{0}\right| \sin \phi$ directly. For $d \neq 0$ the energy spectrum is modified and both positive and negative energy surface states occur in general.

Let us apply the above result to see if the different $x$ 's can be distinguished in $d_{x^{2}-y^{2}}+i x$ superconductors. In Fig. 1 we show the energy gap structure on the $k_{x}-k_{y}$ plane for these superconductors, for $x=s, p, d$. First we consider $d_{x^{2}-y^{2}}+i s$ and $d_{x^{2}-y^{2}}+i d_{x y}$ superconductors. Both superconductors have the property that $\Delta \rightarrow-\Delta^{*}$ upon reflection on the (110) and (1ㅍ) planes, implying absence of zero energy surface states and particle-hole asymmetry in the surface state spectrum for any fixed $k_{F y}$. However, particle-hole symmetry restores in the tunneling spectrum if the experiment samples all $k_{F y}$ vectors equally, since $\Delta \rightarrow-\Delta^{*}$ when $k_{y} \rightarrow-k_{y}$, and the broken time-reversal symmetries in lower and upper $k_{y}$ planes are in opposite domains. After adding the contributions from all $k_{F y}$ 's a particle-hole symmetric spectrum with the zeroenergy bias peak split into two peaks at finite energy will be observed, as predicted in a number of earlier works. ${ }^{8,10}$

The situation is however, different for $d_{x^{2}-y^{2}}+i\left(p_{x} \pm p_{y}\right)$ superconductors. Unlike the $d+i s$ and $d+i d$ states the reflection symmetry of order parameter upon the (110) and (1 $\overline{10})$ planes are different for the $d+i p$ superconductors. $\Delta \rightarrow-\Delta^{*}$ upon reflection on the (110) plane but $\Delta \rightarrow-\Delta$ on the (1 $\overline{10})$ plane or vice versa. Furthermore, the domain of broken timereversal symmetry remains the same $(\Delta \rightarrow-\Delta)$ when $k_{y}$ 
$\rightarrow-k_{y}$. As a result, there will be no $\epsilon=0$ surface states on the (110) [or (1 $1 \overline{1})]$ surface of $d+i p$ superconductors and the corresponding tunneling spectrum, after sampling over all $k_{F y}$ vectors, will remain particle-hole asymmetric, whereas $\epsilon=0$ surface states exist on the (110) [or (110)] surface with particle-hole symmetric tunneling spectrum. The existence of different tunneling spectrums on the (110) and (110) surfaces with one of them being particle-hole asymmetric is a very strong indication of $d+i p$ superconductors. But experiments must be done in monodomain samples where (110) and $(1 \overline{1} 0)$ can be distinguished. There is also the problem that even in a single crystal with a given sign of time reversal, the two different possible reflection domains $\left(p_{x} \pm p_{y}\right)$ may occur. On the other hand, the boundary or tunneling current may pin the orientation of the $p$-order parameter resulting in only one of two possible surface behaviors. Thus if a strong asymmetry is observed in conductance with a peak predominantly for $V>0$ or $V<0$, a bulk $d+i p$ state may be suspected.

For $x=i s, d_{x y}$, one must also consider the possible surface induced pair breaking. ${ }^{8}$ This can be distinguished by the temperature where it sets in, the fact that there is no obvious composition dependence and that its amplitude is expected to be smaller than the bulk effect if the surface layer thickness $l$ is small $\sim O(l \gamma)^{2}$. Otherwise experiments which are sensitive only to the bulk state order parameter have to be considered. We shall examine such experiments in Sec. IV.

\section{EXISTING ANDREEV REFLECTION EXPERIMENTS}

Given the above information let us examine the existing tunneling experiments. Early Andreev reflections experiments on samples of 123-cuprate ${ }^{11}$ with tunneling in the (110) directions have revealed a symmetric peak at zero voltage which split on applying a magnetic field as predicted ${ }^{8}$ for pure $d_{x^{2}-y^{2}}$ symmetry. Subsequently two groups ${ }^{12,13}$ have reported experiments which in zero magnetic field show two peaks with different heights at $\pm \delta V$. The authors correctly conclude that a peak not at zero voltage is an indication of time-reversal-violating superconductivity. Particularly interesting is the fact that in a given tunneling junction, the asymmetry in the amplitude of the peaks increases in annealing up to about a factor of $4 .^{13}$ Further experimental confirmation of the asymmetry on monodomain samples are therefore important because an exact results of our analysis is that the absence of particle-hole symmetry in the tunneling spectrum indicates a bulk time-reversal violating $d+i p$ state. Moreover, differences of opinion ${ }^{14}$ exist about the surface composition of samples and it is not clear where the region of the peaks at zero and nonzero $V$ lie in the phase diagram of 123-cuprates.

\section{SUPERCONDUCTING RINGS WITH MULTIPLE GRAIN BOUNDARY JOSEPHSON JUNCTIONS}

Next consider flux-quantization experiment on superconducting rings with multiple grain boundary Josephson

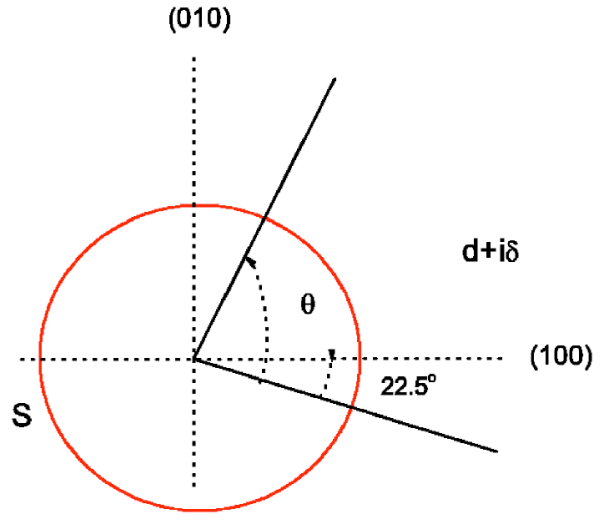

FIG. 2. Arrangement of the two superconducting grains in the proposed two-grain ring. Notice that the (100) axis of the $d+i \delta$-wave superconductor is $22.5^{\circ}$ off the cutting $x$ axis. The flux trapped in the ring changes differently for different superconducting states when $\theta$ increases.

junctions. ${ }^{15,16}$ With suitable arrangement of the grain boundaries these experiments can in principle be used to distinguish between different broken time-reveral symmetry states. We start with a simple ring made of two monocrystal grains as in the Wollman et al. experiments. ${ }^{16}$ One grain is a $s$-wave superconductor, and the other is the $d+i x$-wave superconductor of interests. The arrangement of the two grains is shown in Fig. 2.

The (100) axis of the $d+i x$-wave superconductor is chosen to be $22.5^{\circ}$ off one of the cut directions. Consider the flux quantization condition as a function of the cutting angle $\theta$. From Fig. 1 we see that for $\theta<22.5^{\circ}$ the phases of the $d+i x$ superconductors remain constant for all three choices of $x$ and the flux trapped in the ring is integral multiple of magnetic flux quantum $\Phi_{o}=h / 2 e$, as in usual $s$-wave superconductor rings. For $22.5^{\circ}<\theta<67.5^{\circ}$, the phase of the $d_{x^{2}-y^{2}}+i d_{x y}$ superconductor differs by $2 \phi$ on the two surfaces but remains the same for the other two cases. ${ }^{17}$ Consequently the flux trapped in the ring is $[n \pm(\phi / \pi)] \Phi_{0}$ for the $d+i d$ superconductor, and remains at $n \Phi_{0}$ for the other two kinds. A different regime is reached for $67.5^{\circ}<\theta<112.5^{\circ}$ where the phase difference between the two surfaces of the $d+i d$ superconductor is precisely $\pi$, whereas it is $\pi \pm 2 \phi$ for $d$ $+i$ s superconductor, and can be either $\pi$ or $\pi \pm 2 \phi$ for the $d+i p$ superconductor, depending on the orientation of the $p$-order parameter. Provided that $\phi$ is large enough, the different behaviors of magnetic flux trapped in these different $\theta$ regions can distinguish $d+i d$ superconductor from the other two types. The presence of two different values of trapped flux, if observable, can also distinguish $d+i p$ superconductor from the rest. However the magnetic flux may pin the orientation of the $p$-order parameter, or only one of the two $\left(p_{x} \pm p_{y}\right)$ states may be present. The $d+i p$ wave superconductors can be distinguish from the rest if we further increase $\theta$ to $157.5^{\circ}<\theta<202.5^{\circ}$. In this regime, the phases on the two surfaces of the $d+i p$ superconductor always differ by $2 \phi$ but remain the same for the other two cases. The trapping of nonintegral flux quantum in this region is a clear signature for $d+i p$ order parameter.

Another interesting configuration is to study Josephson effect across two $180^{\circ}$ grains where the cuprate supercon- 
ductor has surfaces pinned at the $\pm(110)$ directions. This direction is gapless for pure $d_{x^{2}-y^{2}}$-wave superconductor but are gapped for $d+i s$ and $d+i d_{x y}$ superconductors. The existence of Josephson effect in this orientation provides unambiguous proof that the superconductor has a mixed order parameter. In this geometry, the $d+i p$ wave superconductor presents an interesting possibility. Since $d+i p$ wave superconductor is gapless along one of the (110) or (110) directions and gapped in the other, Josephson effect with phase shift near $\pi \pm \phi$ is expected in one orientation and no Josephson effect in the other. However there is the possibility that the orientation of the $p$-order parameter may be pinned by the boundary (near a surface with normal $x \pm y$ the $d+i p_{y \mp x}$ state is not permitted since it corresponds to current normal to the surface) plus other experimental limitations which we are not experts on.

Finally, let us study 3-junction rings in the celebrated geometry first fabricated by Tsuei et al. ${ }^{15}$ The ring is built by the superconductor of interests only, but with different crystal orientations in the three grains. On the $k_{x}-k_{y}$ plane the three grains cover regions specified by angles $180^{\circ}-360^{\circ}$, $303.5^{\circ}-360^{\circ}$, and $26.5^{\circ}-150^{\circ}\left[0^{\circ}\right.$ is the (100) axis], respectively (see Fig. 3). For $d$-wave superconductor the phase difference is $\pi$ on the two surfaces of the second grain but the phases are the same on the two surfaces of other grains. Therefore the flux trapped in the ring is precisely $\left(n+\frac{1}{2}\right) \Phi_{0}$.

Repeating the analysis for the $d+i x$ superconductors, we find that the flux trapped in the corresponding rings of $d$ $+i s$ and $d+i d$ superconductors are $F_{s(d)}=\left[n+\frac{1}{2} \pm(\phi / \pi)\right] \Phi_{0}$, whereas the flux trap in the $d+i p$ superconductor can be either $F_{p}=\left(n+\frac{1}{2}\right) \Phi_{0}$ or $\left[n+\frac{1}{2} \pm(\phi / \pi)\right] \Phi_{0}$, depending on the orientation of the $p$-order parameter. Notice that by changing cutting angles the value of the trapped flux will change. For example, if the three grains having cutting angles $180^{\circ}-360^{\circ}, 280^{\circ}-350^{\circ}$, and $60^{\circ}-170^{\circ}$, then the flux trapped in a pure $d$-wave superconductor ring will be $n \Phi_{0}$, whereas the $d+i p$ superconductor will trap flux $F_{p}$ $=[n \pm(\phi / \pi)] \Phi_{0}$, independent of the orientation of the p-order parameter.

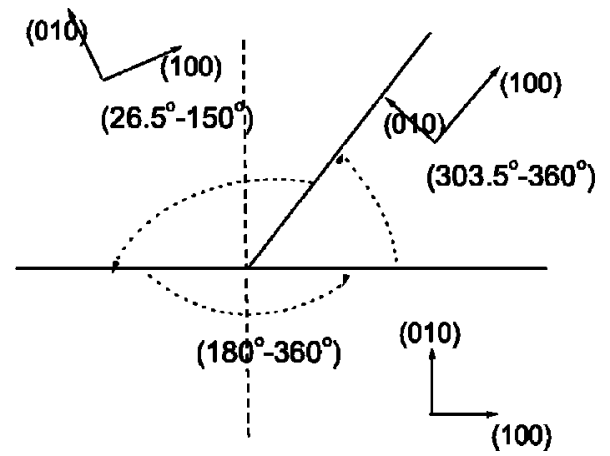

FIG. 3. Arrangement of the three superconducting grains in the experiment by Tsuei et al.

Recently, Tsuei et al. have reported experimental results on superconductor rings in essentially their original 3 -junction ring geometry for three different cuprate systems at various dopings. ${ }^{18}$ They observed that the trapped flux is always $\left(n+\frac{1}{2}\right) \Phi_{0}$ within experimental accuracy of a few percent. Based on this observation, they conclude that the mixing of $d_{x^{2}-y^{2}}+i s$ and $d_{x^{2}-y^{2}}+i d_{x y}$ states, if they exist, must be small in a large range of doping in the cuprates. While we agree with their conclusion, our analysis shows that the $d_{x^{2}-y^{2}}+i\left(p_{x} \pm p_{y}\right)$ state is not ruled out by their results. We therefore suggest experiments with different cutting angles as discussed above, especially in view of the results of Refs. 12 and 13 which suggest time-reversal-violating superconducting state. These experiments may also be more precise since the dominant flux due to the $d$-wave symmetry is nulled out.

\section{ACKNOWLEDGMENTS}

C.M.V. wishes to thank Guy Deutscher, Brigitte Leridon, and Jerome Lesueur for interesting him in this investigation and for various useful comments. T.K.N. acknowledges support of HKRGC through Grant No. 602803.
${ }^{1}$ C. M. Varma, Phys. Rev. B 55, 14554 (1997); Phys. Rev. Lett. 83, 3538 (1999).

${ }^{2}$ M. E. Simon and C. M. Varma, Phys. Rev. Lett. 89, 247003 (2002); Phys. Rev. B 61, R3804 (2000).

${ }^{3}$ A. Kaminski et al., Nature (London) 416, 610 (2002).

${ }^{4}$ M. E. Simon and C. M. Varma, Phys. Rev. B 67, 054511 (2003);

S. Di Matteo and C. M. Varma, ibid. 67, 134502 (2003).

${ }^{5}$ R. P. Kaur and D. F. Agterberg, Phys. Rev. B 68, 100506 (2003).

${ }^{6}$ M. Fogelstrom, D. Rainer, and J. A. Sails, Phys. Rev. Lett. 79, 281 (1997)

${ }^{7}$ R. B. Laughlin, Phys. Rev. Lett. 80, 5188 (1998).

${ }^{8}$ M. Vojta, Y. Zhang, and S. Sachdev, Phys. Rev. B 62, 6721 (2000).

${ }^{9}$ C.-R. Hu, Phys. Rev. Lett. 72, 1526 (1994).

${ }^{10}$ M. Matsumoto and H. Shiba, J. Phys. Soc. Jpn. 64, 4867 (1995).

${ }^{11}$ J. Leseur et al., Physica C 191, 325 (1992); M. Aprili, J. E.
Bedica, and L. H. Greene, Phys. Rev. Lett. 83, 4630 (1999); R. Krupke and G. Deutscher, ibid. 83, 4634 (1999); M. Covington, M. Aprili, E. Paraoanu, L. H. Greene, F. Xu, J. Zhu, and C. A. Mirkin, ibid. 79, 277 (1997).

${ }^{12}$ Y. Dagan and G. Deutscher, Phys. Rev. Lett. 87, 177004 (2001).

${ }^{13}$ X. Grisom, Thesis, Ecole Polytechnic, Paris, 2000.

${ }^{14}$ G. Deutscher, L. Greene, B. Leridon, and J. Leseur (private communication).

${ }^{15}$ C. C. Tsuei et al., Phys. Rev. Lett. 73, 593 (1994); C. C. Tsuei and J. R. Kirtley, Rev. Mod. Phys. 72, 969 (2000).

${ }^{16}$ D. A. Wollman, D. J. Harlingen, J. Giapinkzakis, and D. M. Ginsberg, Phys. Rev. Lett. 74, 797 (1995).

${ }^{17}$ M. R. Beasley, D. Lew, and R. B. Laughlin, Phys. Rev. B 49, 12330 (1994).

${ }^{18}$ C. C. Tsuei et al., cond-mat/0402655 (unpublished). 